MATHEMATICS OF COMPUTATION

Volume 76, Number 259, July 2007, Pages 1483-1492

S 0025-5718(07)01975-8

Article electronically published on March 8, 2007

\title{
ON GENERALIZED AVERAGED GAUSSIAN FORMULAS
}

\author{
MIODRAG M. SPALEVIĆ
}

\begin{abstract}
We present a simple numerical method for constructing the optimal (generalized) averaged Gaussian quadrature formulas which are the optimal stratified extensions of Gauss quadrature formulas. These extensions exist in many cases in which real positive Kronrod formulas do not exist. For the Jacobi weight functions $w(x) \equiv w^{(\alpha, \beta)}(x)=(1-x)^{\alpha}(1+x)^{\beta}(\alpha, \beta>-1)$ we give a necessary and sufficient condition on the parameters $\alpha$ and $\beta$ such that the optimal averaged Gaussian quadrature formulas are internal.
\end{abstract}

\section{INTRODUCTION}

Let $w$ be a given nonnegative and integrable weight function on an interval $[a, b]$. We call an interpolatory quadrature formula (abbreviated q.f.) of the form

$$
\int_{a}^{b} f(x) w(x) d x=Q_{n}[f]+R_{n}[f], \quad Q_{n}[f]=\sum_{j=1}^{n} \omega_{j} f\left(x_{j}\right),
$$

where $x_{1}<x_{2}<\cdots<x_{n}, \omega_{j} \in \mathbb{R}(j=1, \ldots, n)$ and $R_{n}[f]=0$ for $f \in \mathbb{P}_{2 n-m-1}$ $\left(\mathbb{P}_{n}\right.$ denotes as usual the set of polynomials of degree at most $\left.n\right), 0 \leq m \leq n$, a $(2 n-m-1, n, w)$ q.f. If in addition all quadrature weights $\omega_{j}, j=1, \ldots, n$, are positive then it is called a positive $(2 n-m-1, n, w)$ q.f. Furthermore we say that a polynomial $t_{n} \in \mathbb{P}_{n}$ generates a $(2 n-m-1, n, w)$ q.f. if $t_{n}$ has $n$ simple zeros $x_{1}<x_{2}<\cdots<x_{n}, t_{n}(x)=\prod_{j=1}^{n}\left(x-x_{j}\right)$, and if the interpolatory q.f. based on the nodes $x_{j}, j=1, \ldots, n$, is a $(2 n-m-1, n, w)$ q.f. $\mathrm{A}(2 n-m-1, n, w)$ q.f. is internal if all its nodes belong to the closed interval $[a, b]$. A node not belonging to the interval $[a, b]$ is called an exterior node.

Next let us denote by $p_{k}$ the monic polynomial of degree $k$ which is orthogonal to $\mathbb{P}_{k-1}$ with respect to $w$, i.e.

$$
\int_{a}^{b} x^{j} p_{k}(x) w(x) d x=0, j=0,1, \ldots, k-1,
$$

and let us recall that $\left(p_{k}\right)$ satisfies a three-term recurrence relation of the form

$$
p_{k+1}(x)=\left(x-\alpha_{k}\right) p_{k}(x)-\beta_{k} p_{k-1}(x), \quad k=0,1, \ldots,
$$

where $p_{-1}(x)=0, p_{0}(x)=1$ and the $\beta_{k}$ 's have the property to be positive.

Received by the editor August 9, 2005 and in revised form, May 4, 2006.

2000 Mathematics Subject Classification. Primary 65D30, 65D32; Secondary 33A65.

Key words and phrases. Averaged and anti-Gaussian quadrature formula, optimal stratified extension, three-term recurrence relation, positive quadrature formula, Gauss, Jacobi matrix, Kronrod.

The author was supported in part by the Serbian Ministry of Science and Environmental Protection (Project \#144005A: "Approximation of linear operators").

(c) 2007 American Mathematical Society 
The unique q.f. with $l$ nodes and highest possible degree of exactness $2 l-1$ is the Gaussian formula with respect to the weight $w$,

$$
Q_{l}^{G}[f]=\sum_{j=1}^{l} \omega_{j}^{G} f\left(x_{j}^{G}\right) .
$$

As shown by Golub and Welch [8], the nodes of the q.f. $Q_{l}^{G}$ are the eigenvalues, and the weights are proportional to the squares of the first components of the eigenvectors, of the symmetric Jacobi tridiagonal matrix

$$
J_{l}^{G}(w)=\left[\begin{array}{cccc}
\alpha_{0} & \sqrt{\beta_{1}} & & \mathbf{0} \\
\sqrt{\beta_{1}} & \alpha_{1} & \ddots & \\
& \ddots & \ddots & \sqrt{\beta_{l-1}} \\
\mathbf{0} & & \sqrt{\beta_{l-1}} & \alpha_{l-1}
\end{array}\right] .
$$

An important but difficult task in practical calculations is the estimation of the error of the Gaussian q.f. $Q_{l}^{G}[f]$. A typical method, used in most of the standard software libraries, consists of computing a second q.f. with more nodes, typically $2 l+1$, and to use its difference to the Gaussian formula as an error estimate for the Gaussian formula. For economical reasons most often the function values which were used to compute the Gauss q.f. are used again by the $2 l+1$ point formula, such that only $l+1$ new function values have to be considered. Note that for a nontrivial extension of Gaussian formulas, $l+1$ is the minimum number of nodes. Conversely, $l+1$ is a natural number of new nodes, in particular if these interlace with the $l$ nodes of the Gaussian formula. One may consider the $l+1$ new nodes as free parameters and choose them in such a way that the degree of exactness of the $2 l+1$ point formula is as high as possible. This leads to the well-known GaussKronrod q.f. with $2 l+1$ points and degree of exactness at least $3 l+1$. For the Legendre weight function $w(x) \equiv 1$ on $[-1,1]$, and for many other ones on compact intervals, the Gauss-Kronrod q.f. with $2 l+1$ points and degree of exactness at least $3 l+1$, are known to exist, i.e., to have real zeros inside the integration interval that interlace with the nodes of Gaussian formula, and to have positive weights. The polynomial of degree $l+1$ which vanishes at the $l+1$ additional nodes, the so-called Stieltjes polynomial, usually denoted by $E_{l+1}$, is characterized by an orthogonality relation with respect to a sign changing weight. The efficient numerical methods for calculating the positive Gauss-Kronrod q.f. are proposed by Laurie [12, and Calvetti et al. 22 (see also Monegato [13, and Gautschi 6]). But often the weight function $w$ is such that the Gauss q.f. does not possess a real Kronrod extension, e.g. the Gauss-Laguerre and Gauss-Hermite cases [9]. Recently, for the Gegenbauer weight $w^{(\alpha, \alpha)}(x)=\left(1-x^{2}\right)^{\alpha}$, Peherstorfer and Petras [18] showed nonexistence of a Gauss-Kronrod formulae for $l$ sufficiently large and $\alpha>5 / 2$. Analogous results for the Jacobi weight function $w^{(\alpha, \beta)}(x)=(1-x)^{\alpha}(1+x)^{\beta}$ can be found in their paper [19], in particular nonexistence for large $l$ of Gauss-Kronrod formulae when $\min (\alpha, \beta) \geq 0$ and $\max (\alpha, \beta)>5 / 2$.

An interesting approach, initiated by Laurie [10, 11] and Patterson [14], is to construct, for given $\theta \in \mathbb{R}$, a new quadrature formula $Q_{l+1}$ for the functional

$$
I_{\theta}[f]:=\int_{a}^{b} f(x) w(x) d x-\theta Q_{l}^{G},
$$


and to use the so-called stratified quadrature formulas

$$
Q_{2 l+1}=\theta Q_{l}^{G}+Q_{l+1}
$$

for the estimation of the error of $Q_{l}^{G}$. As a special case, the so-called anti-Gaussian formulas $Q_{l+1}^{A}$ were introduced by Laurie [11,

$$
R_{l+1}^{A}\left[x^{k}\right]=-(1+\gamma) R_{l}^{G}\left[x^{k}\right], \quad k=0,1, \ldots, 2 l+1 .
$$

Laurie's definition in [11] is for $\gamma=0$. The more general definition in (1.3) has been used by Ehrich [4] to construct modified formulas. The averaged formula

$$
Q_{2 l+1}^{G A}=\frac{1}{2+\gamma}\left((1+\gamma) Q_{l}^{G}+Q_{l+1}^{A}\right), \quad \gamma>-1,
$$

also introduced in [11] for $\gamma=0$, is of stratified type and has at least the degree of exactness $2 l+1$. In Ehrich [4] the construction of $Q_{2 l+1}^{G A}$ in (1.4), in the sense of a higher degree of exactness, for the Laguerre and Hermite weight functions, has been improved. By construction (he chooses $\gamma$ such that the degree of the extension is increased), these modified averages are also stratified extensions, and among all stratified extensions they are the unique formulas with highest possible degree of exactness. We denote them by $Q_{2 l+1}^{G F}$.

This paper studies the q.f. which is the same as the just quoted optimal (generalized) averaged Gaussian q.f. $Q_{2 l+1}^{G F}$. In the following section we propose the construction of the formula via a $(2 l+1) \times(2 l+1)$ matrix rather than in two stages by an $(l+1) \times(l+1)$ and $l \times l$ matrix. In Section 3 we investigate for which values of $\alpha, \beta$ is the new formula internal in the case of the Jacobi weight function $w^{(\alpha, \beta)}(x)$.

\section{NumericAl CONSTRUCTION}

We study the quadrature formula $Q_{2 l+1}$ obtained as the Gaussian formula arising from the $(2 l+1) \times(2 l+1)$ tridiagonal matrix $J_{2 l+1}^{G F}(w)$ constructed as follows:

(C1) The upper $(l+1) \times(l+1)$ submatrix is the same as the Jacobi matrix for the $(l+1)$-point Gaussian rule for a certain weight $w$, i.e., $J_{l+1}^{G}(w)$.

(C2) The lower $l \times l$ submatrix is the same as the reverse Jacobi matrix for the $l$-point Gaussian rule for $w$,

$$
J_{l}^{*}(w)=\left[\begin{array}{cccc}
\alpha_{l-1} & \sqrt{\beta_{l-1}} & & \mathbf{0} \\
\sqrt{\beta_{l-1}} & \alpha_{l-2} & \ddots & \\
& \ddots & \ddots & \sqrt{\beta_{1}} \\
\mathbf{0} & & \sqrt{\beta_{1}} & \alpha_{0}
\end{array}\right] .
$$

(C3) The remaining codiagonal element is the same as the corresponding element of the Jacobi matrix for the $(l+2)$-point Gaussian rule $J_{l+2}^{G}(w)$.

Therefore,

$$
J_{2 l+1}^{G F}(w)=\left[\begin{array}{ccc}
J_{l+1}^{G}(w) & \sqrt{\beta_{l}} \mathbf{e}_{l} & \mathbf{0} \\
\sqrt{\beta_{l}} \mathbf{e}_{l}^{T} & \alpha_{l} & \sqrt{\beta_{l+1}} \mathbf{e}_{1}^{T} \\
\mathbf{0} & \sqrt{\beta_{l+1}} \mathbf{e}_{1} & J_{l}^{*}(w)
\end{array}\right],
$$

where $\mathbf{e}_{k}$ denotes the $k$ th coordinate vector in $\mathbb{R}^{l}$.

It is well-known (see e.g. [5]) that there is a one-to-one correspondence between Jacobi matrices and quadrature formulae with positive weights. 
This construction causes the resulting $(2 l+1)$-point quadrature rule to have the following properties:

(P1) The degree of exactness is $2 l+2$.

(P2) The nodes of the $l$-point Gaussian rule $Q_{l}^{G}$ for $w$ are a subset of the new formula.

(P3) The weights of these nodes are constant multiples of the original weights.

Therefore, the formula $Q_{2 l+1}$ is identical to the optimal (generalized) averaged Gaussian q.f. $Q_{2 l+1}^{G F}$ first considered by Ehrich [4] (based on a suggestion of Patterson [14]), since:

- Any q.f. that satisfies (C1) and (C2) must necessarily have properties (P2) and (P3), since if $\mathbf{y}$ is an eigenvector of $J_{l}^{G}(w)$, then $\left[\mathbf{y}^{T} ; 0 ; \operatorname{rev}(\mathbf{y})^{T}\right]^{T}$ is an eigenvector of $J_{2 l+1}^{G F}(w)$, with the same eigenvalue.

- (P1) follows from (C1) and (C3) by the degree-revealing property of the Jacobi matrix.

Remark 2.1. The degree of exactness in (P1) is $2 l+3$, if $w$ is an even weight function, i.e., $w(-x)=w(x)$.

The optimal (generalized) averaged Gaussian q.f. $Q_{2 l+1}^{G F}$, as well as the averaged Gaussian q.f. $Q_{2 l+1}^{G A}$ from (1.4) for $\gamma=0$, can also be derived with the aid of Peherstorfer's characterization results in the theory of positive interpolatory q.f. (1.1) which are as follows (see [17, Theorem 3.2] and also [15, 16]):

A polynomial $t_{n}$ generates a positive $(2 n-1-m, n, w)$ q.f. $(0 \leq m \leq n)$ if and only if $t_{n}$ can be generated by a three-term recurrence relation of the form

$$
t_{j+1}(x)=\left(x-\tilde{\alpha}_{j}\right) t_{j}(x)-\tilde{\beta}_{j} t_{j-1}(x), \quad j=0,1, \ldots, n-1,
$$

$t_{-1}(x)=0, t_{0}(x)=1$, with $\tilde{\alpha}_{j} \in \mathbb{R}$ and $\tilde{\beta}_{j}>0$ for $j=0,1, \ldots, n-1$, and with

$\tilde{\alpha}_{j}=\alpha_{j}$ for $j=0,1, \ldots, n-1-\left[\frac{m+1}{2}\right] \quad$ and $\quad \tilde{\beta}_{j}=\beta_{j}$ for $j=0,1, \ldots, n-1-\left[\frac{m}{2}\right]$

and

$$
\operatorname{sgn} t_{j}(a)=(-1)^{j}, t_{j}(b)>0, \quad j=1, \ldots, n,
$$

which is again equivalent to the fact (see the proof of $d) \Longrightarrow a$ ) in [17, Thm. 3.2]) that $t_{n}$ can be represented in the form $(l:=[(m+1) / 2], n \geq 2 l)$

$$
t_{n}=g_{l} p_{n-l}-\tilde{\beta}_{n-l} g_{l-1} p_{n-l-1},
$$

where $g_{l-1}$ and $g_{l}$ are generated by a three-term recurrence relation of the form

$$
g_{j+1}(x)=\left(x-\tilde{\alpha}_{n-1-j}\right) g_{j}(x)-\tilde{\beta}_{n-j} g_{j-1}(x), \quad j=0,1, \ldots, l-1,
$$

$g_{-1}(x)=0, g_{0}(x)=1$, with $\tilde{\alpha}_{n-1-j} \in \mathbb{R}$ and $\tilde{\beta}_{n-j}>0$ for $j=0,1, \ldots, l-1$;

$\tilde{\beta}_{n-l}>0, \tilde{\beta}_{n-l}=\beta_{n-l}$ if $m=2 l-1$; and

$$
\operatorname{sgn} g_{j}(a)=(-1)^{j}, g_{j}(b)>0, \quad j=1, \ldots, l .
$$

Now let us derive the cases under consideration. Let $n=2 l+1$ and put

$$
\begin{aligned}
\tilde{\alpha}_{n-1-j} & =\alpha_{j} \quad \text { and } \quad \tilde{\beta}_{n-j}=\beta_{j} \quad \text { for } j=0,1, \ldots, l-1, \\
\tilde{\beta}_{n-l} & =\beta_{n-l}(m=2 l-1), \quad \text { i.e., } \quad \tilde{\beta}_{n-l}=\beta_{l}(m=2 l),
\end{aligned}
$$

which immediately yields

$$
g_{j} \equiv p_{j}, \quad j=1, \ldots, l .
$$


Conversely putting

$$
g_{l} \equiv p_{l} \quad \text { and } \quad g_{l-1} \equiv p_{l-1},
$$

the relations (2.3) follow. Hence if (2.4) or (2.3) holds, then (2.2) is reduced to

$$
t_{n} \equiv t_{2 l+1}=p_{l} F_{l+1},
$$

where

$$
\begin{aligned}
F_{l+1}(x) & =p_{l+1}(x)-\tilde{\beta}_{l+1} p_{l-1}(x) \\
& =\left(x-\alpha_{l}\right) p_{l}(x)-\bar{\beta}_{l} p_{l-1}(x),
\end{aligned}
$$

where $\bar{\beta}_{l}=\beta_{l}+\tilde{\beta}_{l+1}$.

Thus the optimal (generalized) averaged Gaussian q.f. $Q_{2 l+1}^{G F}$, where $\tilde{\beta}_{l+1}=$ $\beta_{l+1}(m=2 l-1)$,

$$
\begin{aligned}
\int_{a}^{b} f(x) w(x) d x & =Q_{2 l+1}^{G F}[f]+R_{2 l+1}^{G F}[f] \\
Q_{2 l+1}^{G F}[f] & =\sum_{j=1}^{l} \omega_{j}^{G F} f\left(x_{j}^{G}\right)+\sum_{k=1}^{l+1} \widetilde{\omega}_{k}^{G F} f\left(x_{k}^{F}\right),
\end{aligned}
$$

which has at least degree of exactness $2 l+2$, is based on the zeros of $t_{2 l+1}=p_{l} F_{l+1}$. $x_{j}^{G}(j=1, \ldots, l)$ denotes the zeros of $p_{l}$, i.e., the nodes in the corresponding Gauss q.f. $Q_{l}^{G}$, and $x_{k}^{F}(k=1, \ldots, l+1)$ denotes the zeros of $F_{l+1}$. Ehrich [4] showed that this formula is exactly the optimal stratified extension for the Gauss-Laguerre and Gauss-Hermite q.f., in the corresponding cases.

The interpolatory q.f. based on the zeros of $F_{l+1}$ has the form

$$
\int_{a}^{b} f(x) w(x) d x=Q_{l+1}^{F}[f]+R_{l+1}^{F}[f], \quad Q_{l+1}^{F}[f]=\sum_{k=1}^{l+1} \bar{\omega}_{k}^{F} f\left(x_{k}^{F}\right),
$$

and it has the degree of exactness $2 l-1$ since $F_{l+1}$ is orthogonal on $\mathbb{P}_{l-2}$ with respect to $w$.

Using the equalities (1.2) for $k=0,1, \ldots, l-1$, together with (2.5), because of the uniqueness of interpolatory q.f., the nodes $x_{k}^{F}$ and the (positive) weight coefficients $\bar{\omega}_{k}^{F}$ of the q.f. (2.6), we obtain it very easily by the well-known method for the Gauss q.f. (cf. [8]) based on the QR algorithm, and for the following Jacobi matrix

$$
\bar{J}_{l+1}^{F}(w)=\left[\begin{array}{cc}
J_{l}^{G}(w) & \sqrt{\bar{\beta}_{l}} \mathbf{e}_{l} \\
\sqrt{\bar{\beta}_{l}} \mathbf{e}_{l}^{T} & \alpha_{l}
\end{array}\right] .
$$

Note, if $\tilde{\beta}_{l+1}=\beta_{l}(m=2 l)$, i.e., $\bar{\beta}_{l}=2 \beta_{l}$ in (2.5) , the corresponding positive q.f. has at least a degree of exactness $2 l+1$, hence it is the averaged Gaussian q.f. $Q_{2 l+1}^{G A}$ introduced by Laurie [11] $(\gamma=0)$, who obtained it by halving the sum of the Gauss q.f., based on the nodes of $p_{l}$, and the anti-Gauss q.f. $Q_{l+1}^{A}$, based on the nodes of $F_{l+1}$. Recently, Calvetti and Reichel [3] proposed a modification of the anti-Gauss q.f., and showed that the symmetric Gauss-Lobatto q.f. are modified anti-Gauss q.f.

Since $\bar{\beta}_{l}>0$, it is not difficult to show that the zeros of $p_{l}$ and $F_{l+1}$ interlace. Therefore, the inner nodes $x_{k}^{F}(k=2, \ldots, l)$ are in $[a, b]$. 
Example 2.2. Consider the Jacobi weight function $w^{(\alpha, \beta)}(x)$ with $\alpha=1 / 10, \beta=$ $13 / 5$, on $[-1,1]$. Respective Matlab routines $r_{-} j$ acobi.m, for the coefficients in the three-term recurrence relation of the corresponding Jacobi orthogonal polynomials, and gauss.m, for the nodes and weight coefficients in the corresponding Gauss q.f., are downloadable from the Web site

$$
\text { http : //www.cs.purdue.edu/archives/2002/wxg/codes/ }
$$

which contains a suite of many other useful routines, in part assembled as a companion piece to the book in [6].

$$
\begin{aligned}
& -9.686625499734723 e-001 \\
& -9.316692166472302 e-001 \\
& -8.878728134056509 e-001 \\
& -8.349036773445199 e-001 \\
& -7.743502857984884 e-001 \\
& -7.059593891708822 e-001 \\
& -6.309958682484870 e-001 \\
& -5.497023099157536 e-001 \\
& -4.632684854851569 e-001 \\
& -3.722105713938731 e-001 \\
& -2.776956155761358 e-001 \\
& -1.804207250606203 e-001 \\
& -8.152901380092521 e-002 \\
& 1.816291970948764 e-002 \\
& 1.175565290185397 e-001 \\
& 2.157670908809297 e-001 \\
& 3.117686462151886 e-001 \\
& 4.046555992796639 e-001 \\
& 4.935042803199599 e-001 \\
& 5.774329480111343 e-001 \\
& 6.556481067408614 e-001 \\
& 7.273342841018780 e-001 \\
& 7.918514028863188 e-001 \\
& 8.484906865990399 e-001 \\
& 8.967804407043425 e-001 \\
& 9.361603420077345 e-001 \\
& 9.663230344398555 e-001 \\
& 9.869271655228162 e-001 \\
& 9.977311827889372 e-001
\end{aligned}
$$$$
4.439648661211199 \mathrm{e}-006
$$$$
3.966117290264903 e-005
$$$$
1.749978150852832 \mathrm{e}-004
$$$$
5.583868232282013 e-004
$$$$
1.425092005936056 \mathrm{e}-003
$$$$
3.142720776391268 \mathrm{e}-003
$$$$
6.149123770464934 \mathrm{e}-003
$$$$
1.103233081864974 \mathrm{e}-002
$$$$
1.830113296473133 e-002
$$$$
2.861180701173614 \mathrm{e}-002
$$$$
4.222027743844664 \mathrm{e}-002
$$$$
5.958699164188983 e-002
$$$$
8.029503177639899 \mathrm{e}-002
$$$$
1.043666148326018 \mathrm{e}-001
$$$$
1.303863072192636 \mathrm{e}-001
$$$$
1.578805710572899 \mathrm{e}-001
$$$$
1.844044403039764 \mathrm{e}-001
$$$$
2.092174951833064 \mathrm{e}-001
$$$$
2.292146928349319 \mathrm{e}-001
$$$$
2.438732902126057 e-001
$$$$
2.501387802213795 \mathrm{e}-001
$$$$
2.482762745348956 \mathrm{e}-001
$$$$
2.361134134425601 \mathrm{e}-001
$$$$
2.151210788530024 \mathrm{e}-001
$$$$
1.846686923159468 \mathrm{e}-001
$$$$
1.475369227885079 \mathrm{e}-001
$$$$
1.049200118498772 \mathrm{e}-001
$$$$
6.099624959468915 \mathrm{e}-002
$$$$
1.945739390825556 \mathrm{e}-002
$$

The nodes in the increasing order (the first column) and corresponding weight coefficients (the second column) of the corresponding q.f. $Q_{29}^{G F}$ are displayed in the previous table.

As we have seen, the q.f. $Q_{2 l+1}^{G F}$, which has the degree of exactness $2 l+2$, is an extension of the Gauss formula. Nonexistence for large $l$ of Gauss-Kronrod formulae, for the case of the Jacobi weight function considered in Example 2.2. has been recently proved by Peherstorfer and Petras [19]. Using the Matlab routine kronrod.m, which is downloadable from the above-mentioned Web site, we obtain in the considered case $\left(w^{(1 / 10,13 / 5)}(x), l=14\right)$ that the Gauss-Kronrod q.f. does not exist. For $1 \leq l \leq 13$ the Matlab routine kronrod.m generates the corresponding Gauss-Kronrod q.f. 


\section{3. $Q_{2 l+1}^{G F}$ FOR The JACOBI WEIGHT FUnCTIONS}

Since the q.f. $Q_{2 l+1}^{G F}$ under consideration is of particular interest when all its nodes belong to $[a, b]$, we will consider this question in this section. For the classical weights, the three-term recurrence coefficients and the values of the orthogonal polynomials at the end points are explicitly known (see for instance [1]).

The Jacobi weight function $w^{(\alpha, \beta)}(x)=(1-x)^{\alpha}(1+x)^{\beta}$ over $[-1,1]$ with $\alpha, \beta>-1$ will be considered. From (2.5) with $\tilde{\beta}_{l+1}=\beta_{l+1}$, we derive the following condition for $x_{l+1}^{F}$ to be in $[-1,1]$ :

$$
L_{l}^{\alpha, \beta}(1) \geq 1
$$

where we put

$$
L_{l}^{\alpha, \beta}(\cdot)=\frac{p_{l+1}(\cdot)}{\beta_{l+1} p_{l-1}(\cdot)} .
$$

The derivation for $x_{1}^{F}$ is similar.

Using the tables from [1, we obtain

$$
\begin{aligned}
\beta_{l} & =\frac{4 l(\alpha+l)(\beta+l)(\alpha+\beta+l)}{(\alpha+\beta+2 l-1)(\alpha+\beta+2 l)^{2}(\alpha+\beta+2 l+1)}, \\
p_{l}(1) & =\frac{2^{l}\left(\begin{array}{c}
\alpha+l \\
l
\end{array}\right)}{\left(\begin{array}{c}
\alpha+\beta+2 l \\
l
\end{array}\right)},
\end{aligned}
$$

and hence, using (3.2),

$$
L_{l}^{\alpha, \beta}(1)=\frac{(\alpha+l)(\alpha+\beta+l)\left(l+1+\frac{\alpha+\beta}{2}\right)(\alpha+\beta+2 l+3)}{(l+1)(l+\beta+1)\left(l+\frac{\alpha+\beta}{2}\right)(\alpha+\beta+2 l-1)} .
$$

If $l \geq 2$, we have that $\alpha+\beta+2 l-1 \geq \alpha+\beta+3>0$, and hence the denominator in the last fraction is positive, since $\alpha, \beta>-1$.

From (3.3), after some simple but tedious calculation, we obtain

$$
L_{l}^{\alpha, \beta}(1)=1+\frac{A(l, \alpha, \beta)}{(l+1)(l+\beta+1)\left(l+\frac{\alpha+\beta}{2}\right)(\alpha+\beta+2 l-1)},
$$

where

$$
\begin{aligned}
A(l, \alpha, \beta) & =(\alpha+\beta+2 l+1)\left\{(2 \alpha+1) l^{2}+(2 \alpha+1)(\alpha+\beta+1) l\right. \\
& \left.+\frac{1}{2}(\alpha+\beta)[(\alpha+1)(\alpha+\beta+1)+2(\alpha-\beta)]\right\} .
\end{aligned}
$$

Therefore, $L_{l}^{\alpha, \beta}(1) \geq 1$, if $A(l, \alpha, \beta) \geq 0$ is fulfilled, since $\alpha+\beta+2 l+1>0$, if

$$
(2 \alpha+1) l^{2}+(2 \alpha+1)(\alpha+\beta+1) l+\frac{1}{2}(\alpha+\beta)[(\alpha+1)(\alpha+\beta+1)+2(\alpha-\beta)] \geq 0 .
$$




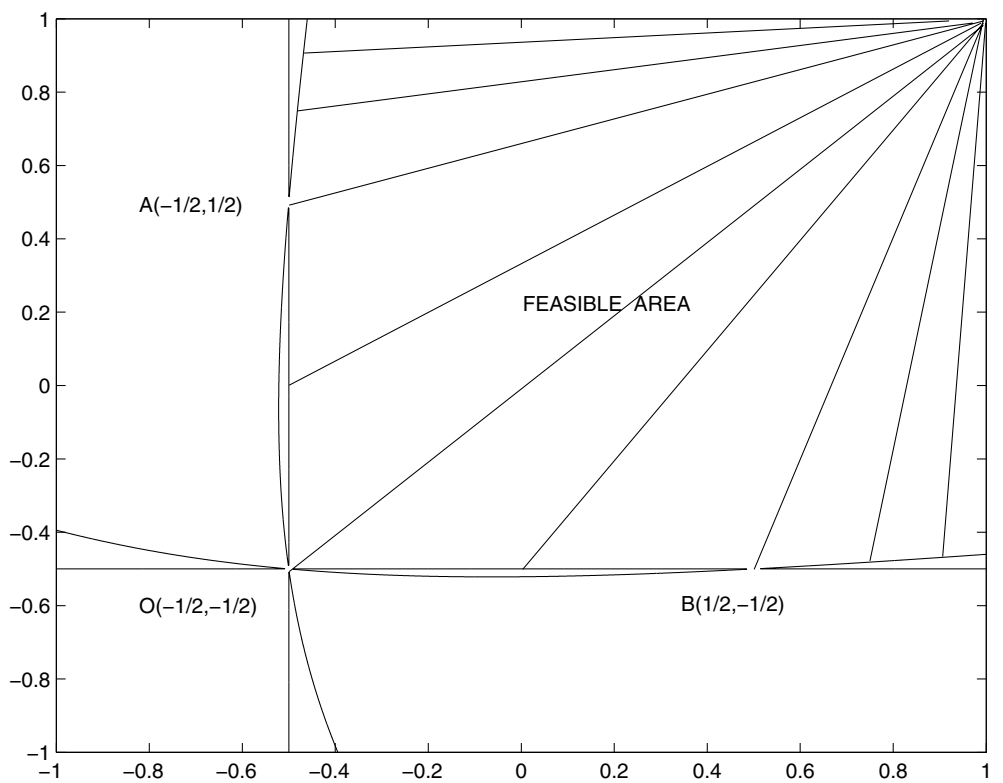

Figure 1. The corresponding optimal averaged Gaussian q.f. $Q_{2 l+1}^{G F}(l \geq 2)$ for the Jacobi weight functions are internal when $\alpha$ and $\beta$ are within the unbounded region to the north-east of the heavy lines, i.e., the region which is lined.

By concluding in an analogous way the point -1 , we obtain the following condition:

$$
(2 \beta+1) l^{2}+(2 \beta+1)(\alpha+\beta+1) l+\frac{1}{2}(\alpha+\beta)[(\beta+1)(\alpha+\beta+1)+2(\beta-\alpha)] \geq 0
$$

Therefore, we have proved the following theorem.

Theorem 3.1. The optimal averaged Gaussian q.f. $Q_{2 l+1}^{G F}$, based on the zeros of the quasi-orthogonal polynomial $p_{l} F_{l+1}$, corresponding to Jacobi weight function $w^{(\alpha, \beta)}(x)=(1-x)^{\alpha}(1+x)^{\beta}$ with $\alpha, \beta>-1$, is internal if and only if the conditions (3.5) and (3.6) hold.

Let us point out that if (3.5) and (3.6) hold for $l=2$, then they hold for all $l \geq 2$. Figure 1 shows the region in the $(\alpha, \beta)$ plane in which the conditions (3.5) and (3.6) are satisfied for $l=2$. Outside that region, the corresponding optimal averaged Gaussian q.f. $Q_{2 l+1}^{G F}$ for at least one value of $l(\geq 2)$ has an exterior node.

Some sufficient conditions for an optimal averaged Gaussian q.f. $Q_{2 l+1}^{G F}$ for the Jacobi weight to require exterior nodes can be deduced from Theorem 3.1. We mention only cases with $\alpha<\beta$ : other cases can be obtained by interchanging $\alpha$ and $\beta$. Denoting the left-hand side of (3.5) by $f(l, \alpha, \beta)$, we have:

1. For $\alpha<-1 / 2$, the formulas for sufficiently large $l$ require an exterior node, because the coefficient of $l^{2}$ is negative.

2. For $\alpha=-1 / 2, \beta>1 / 2(l \geq 2)$, we have $f(l,-1 / 2, \beta)=-3\left(\beta^{2}-1 / 4\right)<0$. 
3. For $\beta>1 / 2$ and $\alpha$ close enough to $-1 / 2(\alpha=-1 / 2+\varepsilon, \varepsilon>0)$, the formulas require an exterior node for $l$ small enough, because $f(l,-1 / 2+\varepsilon, \beta)$ has zeros at

$$
l=\frac{1}{2}\left(-\frac{1}{2}-\beta-\varepsilon \pm \sqrt{\Delta}\right),
$$

where

$$
\Delta=\frac{1}{2}\left(5-3 \varepsilon+3\left(\beta^{2}-1 / 4\right) / \varepsilon\right) .
$$

The positive zero is therefore $O\left(\varepsilon^{-1 / 2}\right)$.

We omit the case when $l=1(\alpha, \beta>-1)$, which can be done easily by the reader.

The cases with Laguerre and Hermite weights are studied by Ehrich [4].

We have used the traditional way of naming the Gauss-Kronrod q.f. although it would have been better to use the name Gauss-Kronrod-Skutsch q.f. (see 7 for details).

\section{ACKNOWLEDGMENTS}

The research was initiated during my visit to Professor Franz Peherstorfer (from Linz University in Austria) in May, 2004. I am thankful to him for introducing me to the theory of positive and Gauss-Kronrod quadrature formulas. I thank both referees for helpful comments concerning the paper.

\section{REFERENCES}

1. M. Abramowitz and I. A. Stegun (eds), Handbook of mathematical functions, National Bureau of Standards, Washington, D.C., 1964. MR0167642 (29:4914)

2. D. Calvetti, G. H. Golub, W. B. Gragg, and L. Reichel, Computation of Gauss-Kronrod rules, Math. Comp. 69 (2000), 1035-1052. MR 1677474 (2000j:65035)

3. D. Calvetti and L. Reichel, Symmetric Gauss-Lobatto and modified anti-Gauss rules, BIT 43 (2003), 541-554. MR2026714 (2004k:65040)

4. S. Ehrich, On stratified extensions of Gauss-Laguerre and Gaus-Hermite quadrature formulas, J. Comput. Appl. Math. 140 (2002), 291-299. MR1934445 (2003g:65027)

5. W. Gautschi, On generating orthogonal polynomials, SIAM J. Scient. Statist. Comput. 3 (1982), 289-317. MR667829 (84e:65022)

6. W. Gautschi, Orthogonal polynomials: computation and approximation, Numerical Mathematics and Scientific Computation, Oxford University Press, Oxford, 2004. MR 2061539 (2005e:42001)

7. W. Gautschi, A historical note on Gauss-Kronrod quadrature, Numer. Math. 100 (2005), 483-484. MR2195449

8. G. H. Golub and J. H. Welsch, Calculation of Gauss quadrature rules, Math. Comp. 23 (1969), 221-230. MR0245201 (39:6513)

9. D. K. Kahaner and G. Monegato, Nonexistence of extended Gauss-Laguerre and GaussHermite quadrature rules with positive weights, Z. Angew. Math. Phys. 29 (1978), 983-986. MR.523866 (80d:65034)

10. D. P. Laurie, Stratified sequences of nested quadrature formulas, Quaestiones Math. 15 (1992), 365-384. MR1192847 (93i:65039)

11. D. P. Laurie, Anti-Gaussian quadrature formulas, Math. Comp. 65 (1996), 739-747. MR 1333318 (96m:65026)

12. D. P. Laurie, Calculation of Gauss-Kronrod quadrature rules, Math. Comp. 66 (1997), 11331145. MR:1422788 (98m:65030)

13. G. Monegato, An overview of the computational aspects of Kronrod quadrature rules, Numer. Algorithms 26 (2001), 173-196. MR.1829797(2002a:65051)

14. T. N. L. Patterson, Stratified nested and related quadrature rules, J. Comput. Appl. Math. 112 (1999), 243-251. MR1728463 
15. F. Peherstorfer, Characterization of positive quadrature formulas, SIAM J. Math. Anal. 12 (1981), 935-942. MR635246 (82m:65021)

16. F. Peherstorfer, Characterization of quadrature formulas II, SIAM J. Math. Anal. 15 (1984), 1021-1030. MR755862 (86a:65025)

17. F. Peherstorfer, On positive quadrature formulas, ISNM, 112, Birkhäuser, Basel, 1993, pp. 297-313. MR1248412 (94k:65035)

18. F. Peherstorfer and K. Petras, Ultraspherical Gauss-Kronrod quadrature is not possible for $\lambda>3$, SIAM J. Numer. Anal. 37 (2000), 927-948. MR1749243 (2001g:33010)

19. F. Peherstorfer and K. Petras, Stieltjes polynomials and Gauss-Kronrod quadrature for Jacobi weight functions, Numer. Math. 95 (2003), 689-706. MR2013124(2004j:33010)

Department of Mathematics and Informatics, University of Kragujevac, Faculty of Science, P.O. Box 60, 34000 Kragujevac, Serbia

E-mail address: spale@kg.ac.yu 